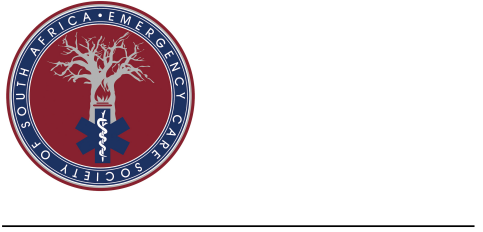

ARTICLE INFORMATION

Received: 16 January 2021

Revision received: 11 April 2021

Accepted: 20 May 2021

Keywords:

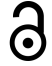

This open access article is distributed under Creative Commons licence CCBY-NC 4.0

ISSN:2709-3786

\section{A proposed definition of Critical Care Retrieval Services in the South African context}

Cornelius Venter ${ }^{1}$, Nathan Conradie ${ }^{1}$, Monique Venter $^{2}$, Louis Jordaan $^{3}$, Maryna Venter ${ }^{4}$, David Stanton ${ }^{2}$, Willem Stassen ${ }^{1, *}$

${ }^{1}$ Division of Emergency Medicine, University of Cape Town

${ }^{2}$ Netcare 911

${ }^{3}$ Department of Emergency Medical Science, Cape Peninsula University of Technology, Cape Town, South Africa

${ }^{4}$ Critical Care Retrieval Services, ER24

On Behalf of the Special Interest Group for Critical Care Retrieval of the Emergency Care Society of South Africa

*Corresponding author: willem.stassen@uct.ac.za, Division of Emergency Medicine,Faculty of Health Sciences, University of Cape Town, Groote Schuur Hospital, Main Rd, Observatory, Cape Town, South Africa

\section{ABSTRACT}

Background: Critical care retrieval is a nascent field in South Africa. An increasing number of private and provincial emergency medical services are starting to invest in developing and setting up dedicated critical care retrieval services (CCRS). To date, there are no minimum standards or definitions on what constitutes a CCRS in the South African context. The aim of this paper is to describe the development of and propose a consensus definition of CCRS within the South African context.

Methods: A literature search was performed to determine the key aspects of CCRS definitions elsewhere. An initial draft definition was circulated to the CCRS working group of the Emergency Care Society of South Africa (ECSSA) for commentary. Comments were collated, and a second round of commentary was undertaken. Finally, a consensus meeting was held through video-conferencing to finalise the definition presented herein. Thereafter, the definition was circulated to key role-players in emergency and critical care, both locally and internationally, for endorsement. The definition, as it is presented, has been endorsed by the ECSSA, the Southern African Society of Critical Care (SASCC), and the International Board for Specialty Certification (IBSC).

Results: The proposed definition outlines five equally important aspects that should be demonstrable and interlinked to be denoted a CCRS. These five aspects are: specific patient populations, case selection, dedicated crew with additional training, dedicated equipment, and continuous quality management and training.

Conclusion: Numerous questions remain unanswered regarding the best approach for CCRS development in South Africa and internationally. Defining what CCRS is in a South African context is a necessary departure point for further research endeavours to develop a core curriculum and practice standards.

Venter C, Conradie N, Venter M, Jordaan L, Venter M, Stanton D, Stassen W. A proposed definition of Critical Care Retrieval Services in the South African context. South African Journal of Pre-hospital Emergency Care. 2021; 2(1):710. doi:10.24213/2-1-4477 


\section{BACKGROUND}

South Africa has relatively few intensive care unit (ICU) beds and medical specialists, especially in peri-urban and rural areas.1 A South African national audit reported 4168 ICU beds were predominantly available in the Gauteng, KwaZulu-Natal and the Western Cape region, with the remaining six provinces only having between 100-300 ICU beds per province. ${ }^{1}$ While there is a paucity of national data relating to the number of critical patients requiring transfer, the shortage of ICU resources implies that the transfer of critically ill or injured patients to facilities more appropriate to manage their conditions is a common occurrence within the South African context. Currently, these transfers are largely undertaken by advanced life support (ALS) prehospital practitioners without additional postgraduate training. ${ }^{2,3}$ The need for additional training and standard-setting for prehospital healthcare practitioners who undertake these transfers has previously been described by the Critical Care Retrieval Working Group (CCRWG). ${ }^{4}$ One of the first steps in standard-setting is to draw a clear distinction between the transfer of a patient from one facility to another, and the transfer of a highly dependent, critical care patient, as this will allow for appropriate resource allocation.

The transfer of patients by practitioners without additional training has been associated with a higher risk of adverse events. ${ }^{5-7}$ In response to this risk, some South African emergency medical services (EMS) have started the process of developing dedicated ALS-based critical care retrieval services (CCRS). Currently, only vague formal regulations exist, and these do not specify aspects such as practitioner training, experience, and minimum equipment standards for dedicated CCRS programmes. ${ }^{6}$

Internationally, CCRS programmes comply with established legislation and adhere to programme or service-specific regulations. ${ }^{8}$ However, the existing legislation and regulations differ in each region or country, and would not always be feasible in the South African context.

Currently, there is no universally accepted South African or international definition for CCRS. An Oxford specialist handbook described retrieval medicine as: "The practice of acute, emergency and critical care medicine in the 'transport environment'". 7 A definition for CCRS will provide a foundation to establish the purpose, structure, and scope for CCRS in South Africa. Literature, which predominantly focuses on the paediatric CCRS patient population, has identified various important key elements that facilitate safe patient transfers. ${ }^{9}$ These elements include the CCRS medical crew selection and team composition, the equipment and mode of transport, which are coordinated by a medical control officer. ${ }^{9}$ The evidence primarily consists of observational studies and discussions focusing on adverse event reduction. ${ }^{10}$

The purpose of this paper is to describe the development of and propose a consensus definition of CCRS within the South African context.

\section{THE DEFINITION DEVELOPMENT PRO- CESS}

A literature search was performed to determine the key aspects of CCRS definitions elsewhere before an initial draft definition was circulated to the CCRWG for commentary. Comments were collated, and a second round of commentary was undertaken. Finally, a consensus meeting was held using video-conferencing to finalise the definition presented herein. Thereafter, the definition was circulated to key role-players in emergency and critical care, both locally and internationally, for endorsement. The definition, as it is presented, has been endorsed by the Emergency Care Society of South Africa (ECSSA), the Southern African Society of Critical Care (SASCC), and the International Board for Specialty Certification (IBSC).

The definition of CCRS is multifactorial, and the safe execution of high acuity transfers cannot simply be reduced to the act of transportation. Further to this, literature has identified that patient safety can only be improved with the development of dedicated CCRS teams functioning as part of an integrated system of care, rather than individuals acting in isolation. ${ }^{9-11}$

It is essential to remember that CCR is still in its infancy in South Africa, and many questions remain as to the best approach to these retrievals and transports, both locally and internationally, ${ }^{2,3}$ However, it is our firm belief that standard-setting is an essential first step. ${ }^{4}$ For this reason, our proposed definition outlines five equally important aspects (Figure 1) that should be demonstrable and interlinked to be denoted a CCRS. These five aspects - specific patient populations, case selection, dedicated crew with additional training, dedicated equipment, and continuous quality management and training - are also the focus of ongoing research by the CCRWG. These aspects are further expanded on to provide an extended, narrative definition for CCRS. 


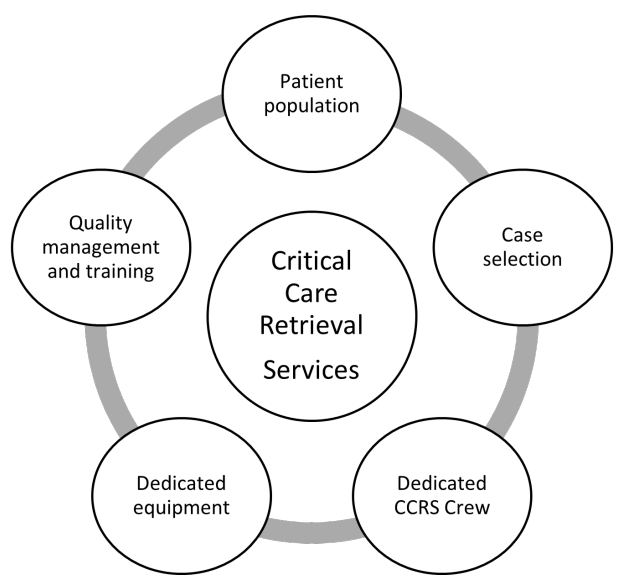

Figure 1: Framework for Defining Critical Care Retrieval Services

\section{Critical Care Retrieval Service: A Definition}

Critical Care Retrieval (CCR) is the stabilisation and transport of a critically ill or injured patient from a location where the patient's healthcare requirements outweigh the diagnostic or treatment abilities, and/or expertise available, to an appropriate facility where these are available. ${ }^{2,11}$

It is acknowledged that defining the term, pragmatically identifying the patient that is "critically ill or injured", and guiding the dispatch of CCRS, is problematic for various reasons. Therefore, a CCRS should have specialised criteria to match the patient population to the CCRS resources. This should be facilitated through a specialised coordinating centre with medical expertise that can evaluate the merits of every case based on clinical and logistical criteria. ${ }^{9}$ Failure to do so may result in considerable variation in dispatch and resource allocation.

A CCRS crew member is a healthcare provider with specific skill, knowledge and expertise matched to the patient population being transported. ${ }^{12}$ These crew members should be in a fulltime CCRS post and should have additional training within the domains of critical care transport. It is essential to bear in mind that this team may be extended to include other medical or technical professionals after assessing the requirements for each case individually. ${ }^{12}$

A CCRS may transport patients via road ambulance, rotor wing or fixed-wing aircraft. Regardless of the mode of transport, these ambulances and aircraft should have dedicated specialised equipment. ${ }^{13}$ Notwithstanding consumables and medications, equipment should, at a minimum, have intensive care monitoring capabilities, ventilation and medication delivery devices; neonatal retrieval would extend these requirements. ${ }^{12}$
A further hallmark of a CCRS is a dedication to continued professional development and ongoing training and certification. It further speaks of a strong clinical governance and quality audit system that allows for dialogue between receiving facilities and specialist teams. ${ }^{12}$ Finally, the system should be responsive to patient safety concerns and proactively prevent adverse events through patient safety systems. ${ }^{14}$

\section{CONCLUSION}

Still a nascent field, numerous questions remain unanswered regarding the best approach for CCRS development in South Africa. Defining CCRS is a necessary departure point for further research endeavours to develop a core curriculum and practice standards.

\section{CONFLICTS OF INTEREST}

The authors report no conflicts of interest.

\section{AUTHOR CONTRIBUTIONS}

Author contributions.

\section{REFERENCES}

1. Bhagwanjee S, Scribante J. National audit of critical care resources in South Africa - unit and bed distribution. S Afr Med J. 2007;97:1311-4.

2. Slabbert J, Smith W. Patient transport from rural to tertiary healthcare centres in the Western Cape: Is there room for improvement? African J Emerg Med. 2011;1:11-6. 
3. Ashokcoomar P, Naidoo R. An analysis of inter-healthcare facility transfer of neonates within the eThekwini Health District of KwaZulu-Natal, South Africa. S Afr Med J. 2017;33:514-8.

4. Venter M, Stanton D, Conradie N, Jordaan L, Venter C, Stassen W. The need for setting standards in critical care transfers. S Afr J Crit Care. 2016;106:32.

5. Wiegersma JS, Droogh JM, Zijlstra JG, Fokkema J, Ligtenberg JJ. Quality of interhospital transport of the critically ill: impact of a Mobile Intensive Care Unit with a specialized retrieval team. Crit Car.e 2011;15:R75.

6. National Health Act No 61 of 2003. Emergency medical services regulations;2017. pp. 74-86.

7. Evans D, Creaton P, Kennedy D, Martin D. Retrieval medicine. New York: Oxford University Press, 2016.

8. National occupational competency profile for paramedics. Paramedic Association of Canada., 2011. (http : / / www - paramedic . ca / uploaded / web / documents / 2011 10 - 31 - Approved - NOCP - English Master.pdf) (visited on 01/11/2021).
9. Ajizian SJ, Nakagawa TA. Interfacility transport of the critically ill pediatric patient. Chest 2007;132:1361-7.

10. Alabdali A, Fisher JD, Trivedy C, Lilford RJ. A systematic review of the prevalence and yypes of adverse events in interfacility critical care transfers by paramedics. 2017.

11. Colyer E, Sorensen M, Wiggins S, Struwe L. The effect of team configuration on the incidence of adverse events in pediatric critical care transport. Air Med J. 2018;37:186-98.

12. Ramnarayan P, Dimitriades K, Freeburn L, et al. Interhospital transport of critically ill children to PICUs in the United Kingdom and Republic of Ireland: Analysis of an international dataset. Pediatr Crit Care Med. 2018;19:e300e311.

13. Craig SS. Challenges in arranging interhospital transfers from a small regional hospital:An observational study. Emerg Med Australas. 2005;17:124-31.

14. Droogh JM, Smit M, Hut J, Vos R de, Ligtenberg JJ, Zijlstra JG. Inter-hospital transport of critically ill patients; expect surprises. Crit Care 2012;16:R26. 